\title{
Image Processing and Analysis for Characterization of Patterns in Polystyrene Foam.
}

\author{
W.A. Heeschen ${ }^{1}$ and V. Woodcraft ${ }^{2}$ \\ ${ }^{1}$ The Dow Chemical Company / Analytical Sciences. Midland, MI \\ 2.The Dow Chemical Company / Building Solutions. Midland, MI
}

Flow-induced irregularities in extrusion-based foaming processes may result in macroscopicallyobserved cell structure patterns in the final products. These resultant inhomogeneities can lead to aesthetic issues, or, in extreme cases, to altered product performance. This report describes characterization of the foam pattern severity via carefully-designed image collection, novel image processing and innovative image analysis. The results were statistically correlated with human observation to create an objective machine vision method for evaluating the foam patterns in a manner consistent with human visual assessment.

The image collection method was designed to be consistent with human perception of the foam pattern. The key element is the minimization of specularly-reflected light which was accomplished using crossed polarization of the incident and analyzed light as shown in Figure 1.

The key component in the analysis is isolation of bright/dark bands due to cumulative effects of many cells in the context of the cell structure wherein 1) individual cell size is on the order of the width of the bands and 2) the cell-by-cell contrast due to the walls and struts is often greater than the apparent contrast from the bands. The result is locally-ambiguous assignment of bright/dark patterns to cells versus bands. The ambiguity was minimized using the "Remove Outliers" option in the ImageJ software ${ }^{i}$ with the parameters tuned to eliminate the bright and dark cell-by-cell outliers in sequential steps (bright noise first, then dark). Individual "noise" pixels which were classified as gray-level outliers relative to the neighborhood were replaced with the median of the neighborhood without the outliers.

The banding pattern features were "Bright" bands, "Dark" bands and "bright/dark transition" bands. The "Bright" and "Dark" band images were processed directly, but the transition bands were identified using the ImageJ Variance convolution filter to highlight the transitions and ignore constant brightness regions ("Variance" bands). The Bright, Dark and Variance band images were then processed with the "Find Ridges" plugini to highlight the bands. The Variance example is shown in Figure 2.

The identified ridges (Bright, Dark and Variance) were kept or rejected for final analysis based on their geometric characteristics. Ridges are either A) lines or B) chevrons (nominal "V" shape). The ridge pattern of interest is radial from the center of the image, so ridges that pointed toward the center of the image were kept and those pointed in other directions were rejected. For lines, the slope and intercept were calculated and if the extended line passed through the center, it was kept. Chevrons were kept if the symmetry centerline of the "V" passed through the center of the image. The centerlines were determined by identifying the two ends, the midpoint in space between the two ends, then the point along the "V" with maximum distance from the two ends and connecting the point of the "V" and the midpoint of the two ends. Figure 3 shows examples of kept/rejected features. 
The total length of the kept features as well as the brightness-weighted lengths were recorded for each of the 83 example images used in this work. Further, each of the 83 images were ranked by nine trained human panelists. The quantitative measurements were statistically reduced to a mathematical model that could be correlated with the panel rankings to determine whether or not the measurements can be used as a direct, objective measure of the patterns. The correlation is plotted in Figure 4 and shows good agreement.

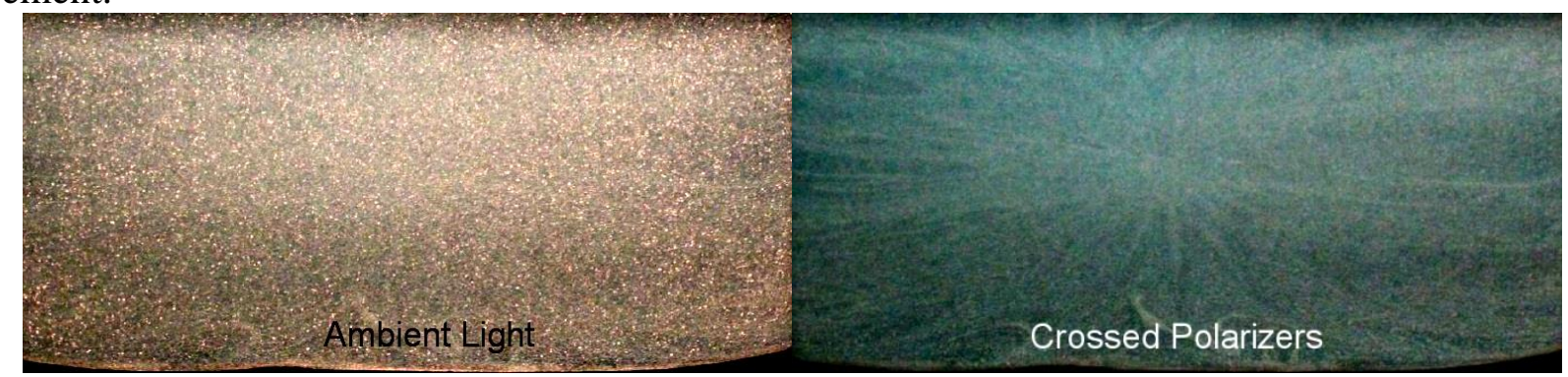

Figure 1. Examples of unpolarized (left) and cross-polarized imaging (right) of the same piece of foam.

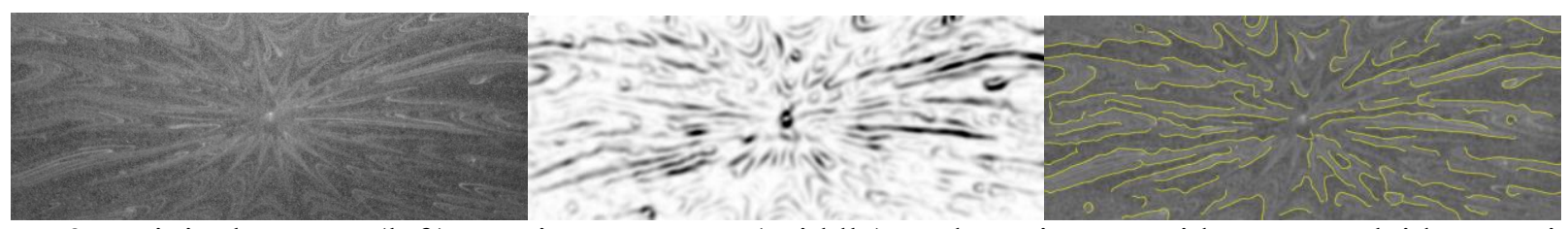

Figure 2. Original Image (left), Variance Image (Middle) and Variance "Ridges" Overlaid on Original (Right).
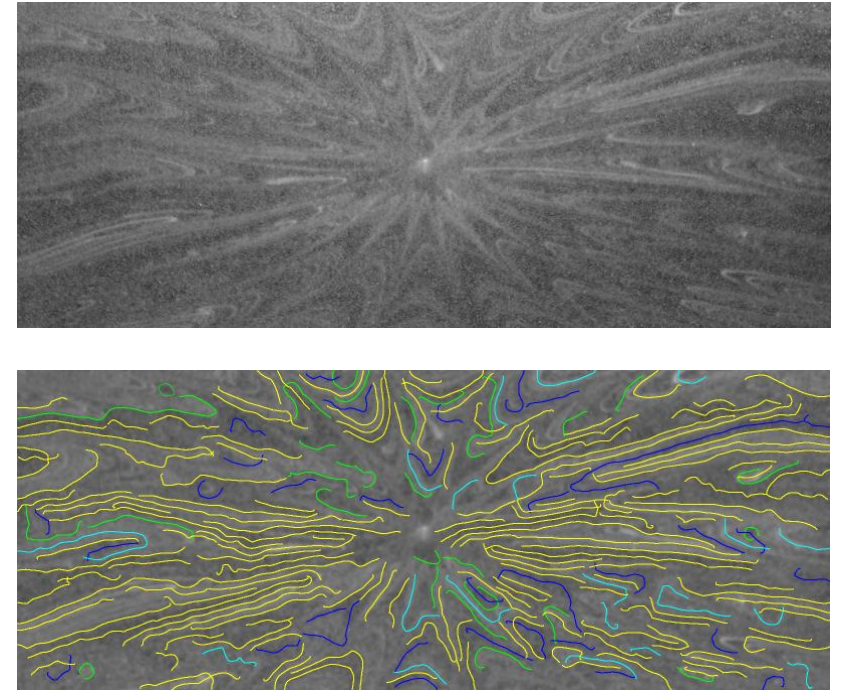

Figure 3. Original Image (Left) and Classified Ridges. Yellow lines are "keepers". Other lines were rejected and are color coded according to their source ridge image: Green=Variance; Cyan=Bright; Blue $=$ Dark.

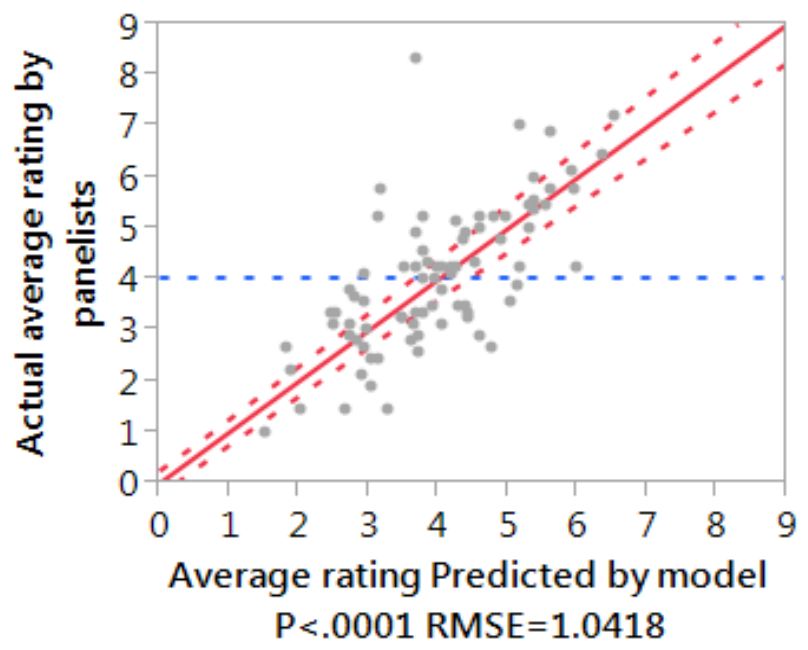

Figure 4. Correlation Between Panelist Rankings and Image Analysis Results. Value of 0 represent little or no patterning; 10 represents strong patterning.

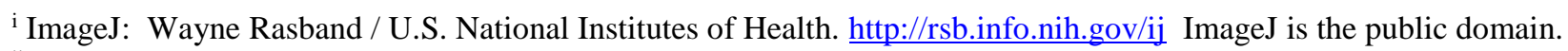

ii Find Ridges plugin by R. Dougherty / Optinav Corp. http://www.optinav.com/imagej.html
} 